RAIRO-Oper. Res. 55 (2021) S481-S502

https://doi.org/10.1051/ro/2019087

RAIRO Operations Research

www.rairo-ro.org

\title{
EQUILIBRIUM JOINING STRATEGIES IN THE SINGLE-SERVER CONSTANT RETRIAL QUEUES WITH BERNOULLI VACATIONS
}

\author{
Ke Sun ${ }^{1}$ And Jinting Wang ${ }^{1,2, *}$
}

\begin{abstract}
We consider the equilibrium joining strategies in an $M / M / 1$ constant retrial queue with Bernoulli vacations. There is no buffer in front of the server, thus an arriving customer will be served immediately if the server is available, and blocked ones wait in a queue if the server is busy or under vacation. The queue length information of orbit is observable to customers upon their arrivals. Then, blocked customers decide whether to join the orbit or not based on a reward-cost structure and their information level. After completing service, the server begins a vacation or remains available and it becomes available again when a vacation ends. The available server seeks to serve the customer in the head of the orbit queue. During the seeking process, an external arrival can interrupt it and obtain service. Our goal is to explore equilibrium behavior of customers in two information cases, fully observable case and almost observable case, which corresponding to whether blocked arrivals can differentiate the state of unavailable server. We obtain the threshold strategies of blocked customers in two information cases and provide numerical experiments to characterize the influence of different parameters on the equilibrium joining strategies.
\end{abstract}

Mathematics Subject Classification. 60K25, 90B22, 91A13.

Received December 11, 2018. Accepted September 6, 2019.

\section{INTRODUCTION}

During the past decades, considerable efforts have been devoted to game-theoretic analysis in various queueing systems. It was initiated by Naor [24] which explored equilibrium and social optimal strategies in an observable $M / M / 1$ queueing model and a reward-cost structure is used to reflect customers' desire for service and unwillingness to wait. Edelson and Hilderbrand [8] extended Naor's model by assuming that arrivals do not have queue length information. Since then, there exists an emerging tendency to derive joining strategies of customers in different queueing systems from economic viewpoint, see Economou and Kanta [6], Burnetas and Economou [4] and among others. Interested readers may refer to the monographs by Hassin and Haviv [13], Tian and Zhang [25] and Hassin [12], they provided comprehensive studies and methodologies in various queueing systems.

As an important part of queueing theory, retrial queue has been extensively studied and it has wide applications in communication networks, cognitive radio networks and telephone switching systems. In retrial queues, customers who find the server is not available upon arrivals are forced to leave the service area and decide

Keywords. Queueing, constant retrial rate, Bernoulli vacations, Nash equilibrium, threshold strategies, partial information.

1 Department of Mathematics, Beijing Jiaotong University, Beijing 100044, PR China.

2 School of Management Science and Engineering, Central University of Finance and Economics, Beijing 100081, PR China.

*Corresponding author: jtwang@cufe.edu.cn 
whether to join a virtual space called orbit or not, then customers in the orbit repeat service requests after random time until succeed. The pioneering work of retrial queues can be found in Cohen [5], Falin [9], Falin and Templeton [10], among others. The constant retrial policy in queue was introduced by Fayolle [11], in which a telephone exchange system was modelled as a retrial queue with FCFS (first-come first-served) discipline in the orbit. Subsequently, more complicated queueing systems with this policy has been studied, see Artalejo [1], Martin and Artalejo [23], Wang et al. [27], and among others. It is quite practical in real life, for example, in communication protocols, the inter-retrial times are controlled by service center. Then, blocked customers may leave their detailed contacts and available server seek customer in in orbit and provide service to the head of the queue.

Besides, queueing systems with vacations have been extensively studied, and the earliest work on vacation queue with Bernoulli scheme was presented by Keilson and Servi [14]. They focused on the system where a sever begins a vacation directly if the system is empty after a service completion, but if there are still customers in the system, it takes a vacation with probability $q$ or continues to work with probability $p(p=1-q)$. Once a vacation ends, the server turns to busy if the queue is not empty; otherwise, it waits for new arrivals. This discipline occurs frequently in real life, in which the server has to operate additional jobs after service such as system maintenance, trouble-saving or just off-the-line periodically. We can find the related applications in multitasking operating systems of computer networks, production and quality control problems, etc., see Kumar and Madheswari [16], Kumar et al. [18], [19], Li et al. [21], and among others.

However, majority of works in vacation queues with retrial policy are devoted to performance analysis and stochastic decomposition, and the game-theoretic analysis is few. The combination of Bernoulli vacation queues with constant retrial policy in queueing analysis can be found in Kumar and Arivudainambi [15], Kumar et al. [17] and Zhou [30]. Moreover, Kulkarni [20] introduced a single-server retrial queue and explored cooperative and noncooperative strategies for two types of customers. Economou and Kanta [7] studied an $M / M / 1$ queue with constant retrial policy and derived customers' equilibrium strategies as well as the social and profit maximization problems. Wang and Zhang [26] investigated equilibrium and socially optimal joining strategies in single-server retrial queue under differen information levels. To learn more equilibrium analysis of constant retrial queue see, for example, Economou and Kanta [7], Zhang et al. [29] and Wang et al. [28]. Liu and Wang [22] considered customers' behavior in constant retrial queue with Bernoulli vacations discipline.

In this study, a single-server constant retrial queue with Bernoulli vacations is analyzed. For example, data transmission is common in network service system, and often they were determined by Transmission Control Protocol (TCP), see the related application in Avrachenkov and Yechiali [2] and [3]. The constant retrial discipline in our paper is a subcase with buffer $N=1$ in [2] and [3], that is, data in transmission which find busy server will join and wait in a queue in the orbit, then the head of the data repeats service request with an exponential rate $\theta$. In addition, virus scanning is a necessary maintenance method to keep the function of the system well, after each data processing, the server takes a vacation to do virus scanning with probability $q$ and resumed to be available with probability $p(p=1-q)$. To the best of the authors' knowledge, studies of the equilibrium behavior of blocked customers in constant retrial queue with Bernoulli vacations do not yet exist so far. Therefore, it is the aim of the present paper to study the strategic behavior of arrivals under different levels of information, where the information refers to the state of unavailable server.

The rest of this paper is structured as follows. Section 2 briefly describes the model and gives some preliminary notations. In Section 3, we derive the corresponding equilibrium threshold strategies of arrivals in the fully observable case and the almost observable case. Section 4 presents some numerical examples to show the effect of information and parameters on the customers' equilibrium behavior, and finally, some conclusions are presented in Section 5.

\section{MODEL DESCRIPTION}

We consider a single-server constant retrial queue, where the server takes vacations with Bernoulli schedule after each service. That is, after each service completion, the server continues to be available with probability 
$p$ and takes a vacation with probability $q$, where $p+q=1$ (we adopt $p \in(0,1)$ to rule out trivial cases). Each vacation period is exponentially distributed with parameter $\gamma$, after vacation, the server becomes available. Potential customers arrive in Poisson process with rate $\lambda$, and the service rate is exponentially distributed with $\mu$. Customers find available server upon arrivals get service immediately and there is no waiting space for blocked arrivals (who find unavailable server, busy or under vacation). These blocked customers are forced to leave the service area, and then decide whether to join an infinite retrial orbit by leaving their contact details or not. The idle server seeks the head customer in the orbit with an exponential rate $\theta$ and provides service to him if he was searched before an external arrival. Therefore, blocked customers in the orbit are served according to FCFS (first-come first-served) discipline. Finally, we assume that the inter-arrival times, service times, vacation times and retrial times are mutually independent.

We denote the system state by a pair $(N(t), I(t))$ at time $t$, where $N(t)$ is the number of customers in the orbit at time $t$, and $I(t)$ represents the state of the server at time $t$ (0: under vacation; 1 : busy; 2 : available). In the fully observable case, arriving customers can observe both $N(t)$ and $I(t)$ whereas in the almost observable case, blocked customers can only observe $N(t)$. Accordingly, the stochastic process $\{(N(t), I(t)), t \geq 0\}$ is a twodimensional continuous time Markov chain with state space $\Omega=\{0,1,2, \ldots\} \times\{0,1,2\}$ and the main transition rates are given for $n=0,1,2, \ldots$ as follows:

$$
\begin{aligned}
p_{(n, i)(n+1, i)} & =\lambda, \quad i=0,1 ; & & p_{(n, 1)(n, 2)}=p \mu ; \\
p_{(n, 1)(n, 0)} & =q \mu ; & & p_{(n, 2)(n, 1)}=\lambda ; \\
p_{(n+1,2)(n, 1)} & =\theta ; & & p_{(n, 0)(n, 2)}=\gamma .
\end{aligned}
$$

We are interested in deriving blocked customers' joining behavior under different information levels, where the information refers to the specific state of an unavailable server (busy or vacation). To model this decision process, we assume that a reward $R$ will be received by each customer after service completion, also, a waiting cost is accumulated at $C$ per unit time before leaving the system (both in the orbit and the server). We consider homogenous and risk neutral customers here, thus $R$ and $C$ are same for all customers, and they concentrate on their own expected utilities based on a linear reward-cost structure (service reward minus waiting cost). In addition, the join/balk decision is irrevocable, i.e., no customer can renege after joining the orbit and re-enter after balking.

\section{Equilibrium threshold STRATEGIES}

In this section, we intend to investigate the existence of customers' equilibrium strategies with threshold type under two information cases. In any case, external arrivals can be served directly when server is available $(I(t)=$ 2 ), thus we focus our research on unavailable server $(I(t)=0,1)$. In the fully observable case, blocked customers observe the number of customers in the orbit and the sate of unavailable server. We demonstrate that the pure threshold strategy is specified by a pair $\left(n_{e}(0), n_{e}(1)\right)$, where blocked customers join if $N(I(t)) \leq n_{e}(I(t))-1$ at $t$ and balks otherwise. In the almost observable case, customers' decisions depend only on $N(t)$ they observe. Thus there exists a single threshold $n_{e}$, where arrivals join if $N(t) \leq n_{e}-1$ at $t$ and balks otherwise. Besides, the condition $R-\frac{C}{\mu}>0$ is necessary, or no customer will join the system even he can get service upon arrival. The stationary distribution of the system under two information cases are studied and the corresponding equilibrium joining behavior are given in the following.

\subsection{Fully observable queue}

In this case, blocked customers receive both the information of server's state and the number of customers in the orbit upon arrivals, and we demonstrate the existence and uniqueness of the pair thresholds $\left(n_{e}(0), n_{e}(1)\right)$. The corresponding transition rate diagram is shown in Figure 1. 


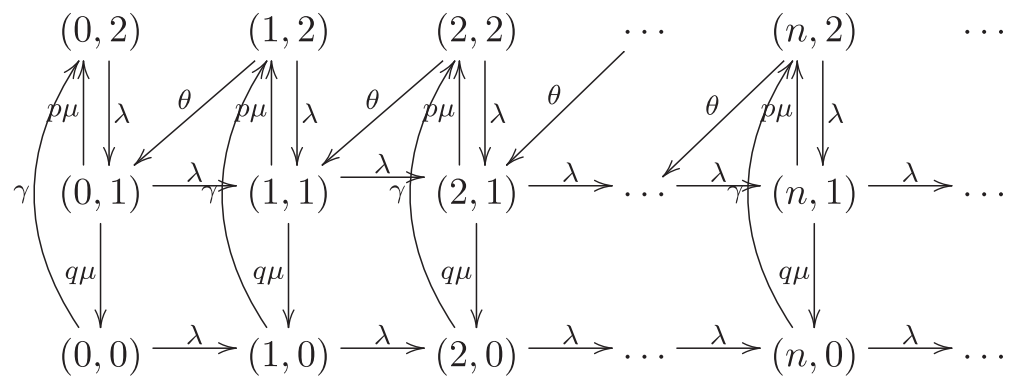

FIGURE 1. State transition diagram of constant retrial queue with Bernoulli vacations.

Theorem 3.1. In the fully observable $M / M / 1$ constant retrial queue with Bernoulli vacations, there exists a pair of thresholds strategy for blocked customers upon arrivals, where

$$
\left(n_{e}(0), n_{e}(1)\right)=\left(\left\lfloor\frac{\mu \theta(\gamma R-p C)}{(\mu \gamma+(q \mu+\gamma)(\lambda+\theta)) C}\right\rfloor,\left\lfloor\frac{\theta \gamma(\mu R-C)}{(\mu \gamma+(q \mu+\gamma)(\lambda+\theta)) C}\right\rfloor\right),
$$

and they all follow the strategy "join the orbit if $N(I(t)) \leq n_{e}(I(t))-1$ and balks otherwise".

Proof. We denote $T(n, i)$ as the expected waiting time in the orbit, given that he is in the $n$th position and the server state is $i(n \geq 1, i=0,1)$. Then we have the following equations:

$$
\begin{array}{rlr}
T(1,0) & =\frac{1}{\gamma}+T(1,2), & \\
T(1,1) & =\frac{1}{\mu}+p T(1,2)+q T(1,0), & \\
T(1,2)=\frac{1}{\lambda+\theta}+\frac{\lambda}{\lambda+\theta} T(1,1), & n=2,3, \ldots, \\
T(n, 0)=\frac{1}{\gamma}+T(n, 2), & n=2,3, \ldots, \\
T(n, 1)=\frac{1}{\mu}+p T(n, 2)+q T(n, 0), & n=2,3, \ldots \\
T(n, 2)=\frac{1}{\lambda+\theta}+\frac{\lambda}{\lambda+\theta} T(n, 1)+\frac{\theta}{\lambda+\theta} T(n-1,1), &
\end{array}
$$

Solving these equations by iteration, we obtain the general formulas of $T(n, i)$ :

$$
\begin{array}{rlrl}
T(n, 0) & =\frac{[\mu \gamma+(q \mu+\gamma)(\lambda+\theta)] n}{\mu \theta \gamma}+\frac{p \mu-\gamma}{\mu \gamma}, & n=1,2,3, \ldots, \\
T(n, 1)=\frac{[\mu \gamma+(q \mu+\gamma)(\lambda+\theta)] n}{\mu \theta \gamma}, & n=1,2,3, \ldots, \\
T(n, 2)=\frac{[\mu \gamma+(q \mu+\gamma)(\lambda+\theta)]) n}{\mu \theta \gamma}-\frac{q \mu+\gamma}{\mu \gamma}, & n=1,2,3, \ldots
\end{array}
$$

Based on the reward-cost structure, the expected net benefit of a blocked customer who decides to join is:

$$
S(n, i)=R-C\left(T(n+1, i)+\frac{1}{\mu}\right),
$$

given that he observes system state $(n, i)(n \geq 0, i=0,1)$ upon arrival. 
If $S(n, i)>0$, i.e., the reward for service completion exceeds the expected waiting cost, he prefers to join, and if $S(n, i)=0$, he is indifferent between joining and balking and balks directly if $S(n, i)<0$. Combining equations (3.2) and (3.3), the mean waiting time when he observes $(n, i)$ is $T(n+1, i)+\frac{1}{\mu}(i=0,1)$ and it is increasing in $n$, thus $S(n, i)$ is monotonically decreasing in $n$.

Therefore, there exists unique threshold which satisfies the conditions $S(n-1, i) \geq 0$ and $S(n, i)<0$ for every $i=0,1$, and we denote it as $n_{e}(I(t))$. Blocked customer enters if $N(I(t)) \leq n_{e}(I(t))-1$ and balks otherwise, which leads to the maximal number of customers in the orbit is $n_{e}(I(t))$. Then, by solving $S(n-1, i)=0$ with the help of equations (3.2) and (3.3), we immediately obtain the value of $n_{e}(I(t))$ in (3.1).

In almost all previous queueing literature with vacations, arriving customers prefer to join the busy server than it is under vacations. However, this preference is not always found in our model by comparing the value of $n_{e}(0)$ and $n_{e}(1)$ in (3.1). This is because that blocked arrivals join the orbit need to wait the server becomes available $(i=2)$ and seeks them, they are more concerned about how long the server state turns to available. Hence, we discuss the order of the two thresholds in state 0 and 1 and find that it depends on the transfer rate from unavailable server to the available one.

Proposition 3.2. In the fully observable $M / M / 1$ constant retrial queue with Bernoulli vacations, the thresholds $n_{e}(0)$ and $n_{e}(1)$ have the following characteristics:

(I) If $p \mu \leq \gamma$, then $n_{e}(1) \leq n_{e}(0) \leq n_{e}(1)+1$;

(II) If $p \mu>\gamma$, then $n_{e}(1)>n_{e}(0)$;

(IIa) when $A+B \geq 0$, we have $n_{e}(1)-n_{e}(0)=1$,

(IIb) when $A+B<0$, we have $n_{e}(1)-n_{e}(0) \geq 1$.

The notations $A, B$ are defined as $A=\theta(\gamma-p \mu), B=\mu \gamma+(q \mu+\gamma)(\lambda+\theta)$.

Proof. From Theorem 3.1, we obtain the specific forms of $n_{e}(1), n_{e}(0)$ and the following equation:

$$
\frac{\mu \theta(\gamma R-p C)}{(\mu \gamma+(q \mu+\gamma)(\lambda+\theta)) C}-\frac{\theta \gamma(\mu R-C)}{(\mu \gamma+(q \mu+\gamma)(\lambda+\theta)) C}=\frac{\theta(\gamma-p \mu)}{\mu \gamma+(q \mu+\gamma)(\lambda+\theta)} .
$$

We denote $A=\theta(\gamma-p \mu), B=\mu \gamma+(q \mu+\gamma)(\lambda+\theta)>0$, where $A-B=-\lambda(q \mu+\gamma)-\mu(\theta+\gamma)<0$. It followed by the three cases below:

(1) If $p \mu<\gamma$, then $n_{e}(0) \geq n_{e}(1)$ and $0<A<B$, then $0<\frac{A}{B}<1, n_{e}(0)-n_{e}(1) \in\{0,1\}$;

(2) If $p \mu=\gamma$, then $A=0$ and $\frac{A}{B}=0$, thus $n_{e}(0)=n_{e}(1)$;

(3) If $p \mu>\gamma$, then $A<0<B$ and $n_{e}(0)<n_{e}(1)$. When $-1 \leq \frac{A}{B}<0, n_{e}(1)-n_{e}(0)=1$, while if $\frac{A}{B}<-1$, then value of the difference between $n_{e}(1)$ and $n_{e}(0)$ is not less than 1 .

These three cases above can be summed up as two main situations in the Proposition 3.2.

Based on Proposition 3.2, the order of $n_{e}(0)$ and $n_{e}(1)$ mainly depends on the difference between $p \mu$ and $\gamma$. Hence we divide the stationary distribution analysis of the system into two parts according to the order of $n_{e}(0)$ and $n_{e}(1)$, and conclude the results in the following two propositions. 


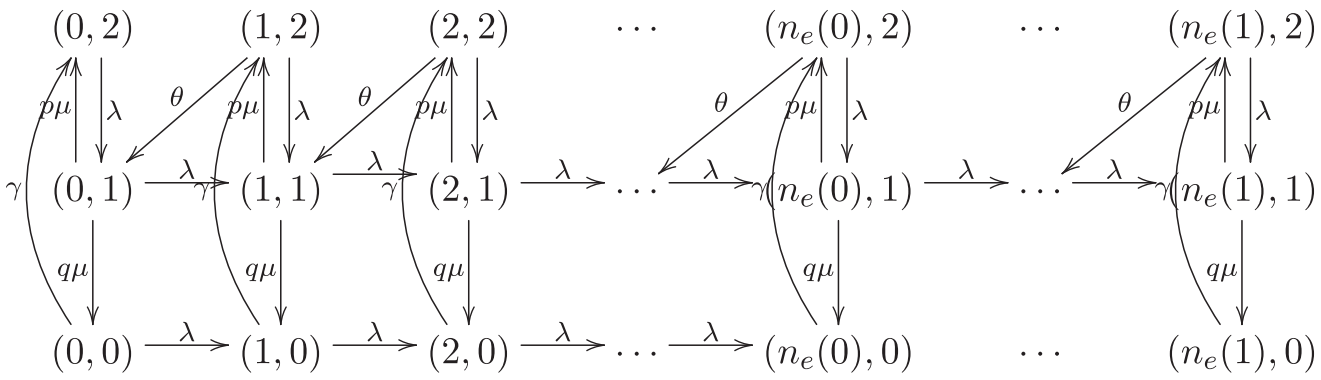

Figure 2. State transition diagram of the fully observable case when $n_{e}(0)<n_{e}(1)$.

Proposition 3.3. In the fully observable $M / M / 1$ constant retrial queue with Bernoulli vacations, when $n_{e}(0)<$ $n_{e}(1)$, the stationary distribution $\left(p^{f}(n, i):(n, i) \in\left\{0,1,2, \ldots, n_{e}\right\} \times\{0,1,2\}\right)$ are given as

$$
\begin{array}{rlrl}
p^{f}(n, 0) & =\widetilde{c}_{1} \rho_{1}^{n}+\widetilde{c}_{2} \rho_{2}^{n}+\widetilde{c}_{3} \rho_{3}^{n}, & & n=0,1, \ldots, n_{e}(0)-1, \\
p^{f}(n, 1) & =\widetilde{\nu}_{1} \rho_{1}^{n}+\widetilde{\nu}_{2} \rho_{2}^{n}+\widetilde{\nu}_{3} \rho_{3}^{n}, & & n=0,1, \ldots, n_{e}(0)-1, \\
p^{f}(n, 2) & =\widetilde{\omega}_{1} \rho_{1}^{n}+\widetilde{\omega}_{2} \rho_{2}^{n}+\widetilde{\omega}_{3} \rho_{3}^{n}, & & n=0,1, \ldots, n_{e}(0)-1, \\
p^{f}\left(n_{e}(0), 0\right) & =\frac{\lambda q(\lambda+\theta)}{\theta \gamma} \widetilde{Y}+\frac{\lambda^{2} q+\lambda \theta}{\theta \gamma} \widetilde{X}, & & \\
p^{f}\left(n_{e}(0), 1\right) & =\frac{\lambda(\lambda+\theta)}{\theta \mu} \widetilde{Y}+\frac{\lambda^{2}}{\theta \mu} \widetilde{X}, & & \\
p^{f}\left(n_{e}(0), 2\right) & =\frac{\lambda}{\theta} \widetilde{Y}+\frac{\lambda}{\theta} \widetilde{X}, \\
p^{f}(n, 0) & =\frac{q \mu}{\gamma}\left(\frac{\theta \mu}{\lambda(\lambda+\theta)}\right)^{n_{e}(0)-n}\left(\frac{\lambda(\lambda+\theta)}{\theta \mu} \widetilde{Y}+\frac{\lambda^{2}}{\theta \mu} \widetilde{X}\right), & & n=n_{e}(0)+1, \ldots, n_{e}(1) \\
p^{f}(n, 1) & =\left(\frac{\theta \mu}{\lambda(\lambda+\theta)}\right)^{n_{e}(0)-n}\left(\frac{\lambda(\lambda+\theta)}{\theta} \widetilde{Y}+\frac{\lambda^{2}}{\theta \mu} \widetilde{X}\right), & & n=n_{e}(0)+1, \ldots, n_{e}(1), \\
p^{f}(n, 2) & =\frac{\mu}{\lambda+\theta}\left(\frac{\theta \mu}{\lambda(\lambda+\theta)}\right)^{n_{e}(0)-n}\left(\frac{\lambda(\lambda+\theta)}{\theta \mu} \widetilde{Y}+\frac{\lambda^{2}}{\theta \mu} \widetilde{X}\right), & & n=n_{e}(0)+1, \ldots, n_{e}(1)
\end{array}
$$

where

$$
\begin{array}{rlr}
\widetilde{\nu}_{i} & =\frac{(\lambda+\gamma) \rho_{i}-\lambda}{q \mu \rho_{i}} \widetilde{c}_{i}, & i=1,2,3, \\
\widetilde{\omega}_{i} & =\frac{(\lambda+\mu) \rho_{i}-\lambda}{\left(\lambda+\theta \rho_{i}\right) \rho_{i}} \widetilde{\nu}_{i}, & i=1,2,3 \\
a & =-\frac{(\lambda+\mu)(\lambda+\gamma)(\lambda+\theta)-\lambda \mu(\lambda p+\gamma)+\lambda p \theta \mu}{\mu \theta(\lambda p+\gamma)}, & \\
b & =\frac{\lambda\{(\lambda+\theta)(2 \lambda+\mu+\gamma)-\lambda p \mu\}}{\mu \theta(\lambda p+\gamma)}, \\
c & =-\frac{\lambda^{2}(\lambda+\theta)}{\mu \theta(\lambda p+\gamma)}, \\
\widetilde{X} & =\widetilde{c}_{1} \rho_{1}^{n_{e}(0)-1}+\widetilde{c}_{2} \rho_{2}^{n_{e}(0)-1}+\widetilde{c}_{3} \rho_{3}^{n_{e}(0)-1}, \\
\widetilde{Y} & =\widetilde{\nu}_{1} \rho_{1}^{n_{e}(0)-1}+\widetilde{\nu}_{2} \rho_{2}^{n_{e}(0)-1}+\widetilde{\nu}_{3} \rho_{3}^{n_{e}(0)-1} .
\end{array}
$$


The coefficient $\widetilde{c}_{i}(i=1,2,3)$ were solved by using normalization condition and $\rho_{i}(i=1,2,3)$ are three roots of equation $x^{3}+a x^{2}+b x+c=0$.

Proof. In this situation, the state transition diagram is shown in Figure 2, and the balance equations are given as

$$
\begin{array}{rlrl}
(\lambda+\gamma) p^{f}(0,0) & =q \mu p^{f}(0,1), & & n=1,2, \ldots, n_{e}(0)-1, \\
(\lambda+\gamma) p^{f}(n, 0) & =\lambda p^{f}(n-1,0)+q \mu p^{f}(n, 1), & & \\
\gamma p^{f}\left(n_{e}(0), 0\right) & =\lambda p^{f}\left(n_{e}(0)-1,0\right)+q \mu p^{f}\left(n_{e}(0), 1\right), & \\
\gamma p^{f}(n, 0) & =q \mu p^{f}(n, 1), \quad n=n_{e}(0)+1, \ldots, n_{e}(1), & \\
(\lambda+\mu) p^{f}(0,1) & =\lambda p^{f}(0,2)+\theta p^{f}(1,2), & & \\
(\lambda+\mu) p^{f}(n, 1) & =\lambda p^{f}(n-1,1)+\lambda p^{f}(n, 2)+\theta p^{f}(n+1,2), n_{e}(1)-1, \\
\mu p^{f}\left(n_{e}(1), 1\right) & =\lambda p^{f}\left(n_{e}(1)-1,1\right)+\lambda p^{f}\left(n_{e}(1), 2\right), & & \\
\lambda p^{f}(0,2) & =\gamma p^{f}(0,0)+p \mu p^{f}(0,1), & n=1,2, \ldots, n_{e}(1) .
\end{array}
$$

The stationary distribution $\left\{p^{f}(n, i):(n, i) \in \Omega\right\}$ is the unique positive solution of the balance equations above. Combining with (3.15), (3.19) and (3.22), we have that

$$
\begin{aligned}
& -\mu \theta(\lambda p+\gamma) p^{f}(n+1,0)+\{(\lambda+\mu)(\lambda+\gamma)(\lambda+\theta)-\lambda \mu(\lambda p+\gamma)+\lambda p \theta \mu\} p^{f}(n, 0) \\
& -\lambda\{(\lambda+\theta)(2 \lambda+\mu+\gamma)-\lambda p \mu\} p^{f}(n-1,0)+\lambda^{2}(\lambda+\theta) p^{f}(n-2,0)=0, n=2, \ldots, n_{e}(0)-2,
\end{aligned}
$$

which is a three-order difference equation with solutions $\rho_{i}(i=1,2,3)$. Therefore, we can set

$$
p^{f}(n, 0)=\widetilde{c}_{1} \rho_{1}^{n}+\widetilde{c}_{2} \rho_{2}^{n}+\widetilde{c}_{3} \rho_{3}^{n}, \quad n=0,1, \ldots, n_{e}(0)-1,
$$

where $\widetilde{c}_{i}(i=1,2,3)$ are undetermined constants we are going to explore next. By plugging (3.51) in (3.15), we have that

$$
p^{f}(n, 1)=\widetilde{\nu}_{1} \rho_{1}^{n}+\widetilde{\nu}_{2} \rho_{2}^{n}+\widetilde{\nu}_{3} \rho_{3}^{n}, \quad n=1,2, \ldots, n_{e}(0)-1 .
$$

Again, by plugging equations (3.51) and (3.25) in (3.22), we have

$$
p^{f}(n, 2)=\widetilde{\omega}_{1} \rho_{1}^{n}+\widetilde{\omega}_{2} \rho_{2}^{n}+\widetilde{\omega}_{3} \rho_{3}^{n}, \quad n=1,2, \ldots, n_{e}(0)-1 .
$$

By ordering $n=1$ in (3.19) and using (3.25) and (3.26), it is followed that:

$$
p^{f}(0,1)=\widetilde{\nu}_{1}+\widetilde{\nu}_{2}+\widetilde{\nu}_{3} .
$$

Then we have (3.6) by combining (3.25) and (3.27). In a similar way, ordering $n=1$ and in (3.22), and from the equations above, we get (3.7) with the help of (3.23) and (3.26). Then, by substituting (3.15) into (3.17), we have

$$
\theta \mu p^{f}(n+1,1)-\left(\lambda^{2}+\lambda \theta+\mu \theta\right) p^{f}(n, 1)+\lambda(\lambda+\theta) p^{f}(n-1,1)=0, \quad n=n_{e}(0)+1, \ldots, n_{e}(1),
$$

which can be considered as a homogeneous linear difference equation and the characteristic equation is

$$
\theta \mu x^{2}-\left(\lambda^{2}+\lambda \theta+\mu \theta\right) x+\lambda(\lambda+\theta)=0, \quad n=n_{e}(0)+1, \ldots, n_{e}(1),
$$

It has two roots, 1 and $\frac{\lambda(\lambda+\theta)}{\theta \mu}$, where the probability $p^{f}(n, 1)$ can be denoted by

$$
p^{f}(n, 1)=\widetilde{A} 1^{n}+\widetilde{B}\left(\frac{\lambda(\lambda+\theta)}{\theta \mu}\right)^{n}, \quad n=n_{e}(0)+1, \ldots, n_{e}(1),
$$


and $\widetilde{A}, \widetilde{B}$ in (3.28) are constants we have to solve. By calculation, we have that

$$
\begin{aligned}
& p^{f}\left(n_{e}(1)-1,1\right)=\widetilde{A}+\widetilde{B}\left(\frac{\lambda(\lambda+\theta)}{\theta \mu}\right)^{n_{e}(1)-1}=\frac{\theta \gamma}{\lambda q(\lambda+\theta)} p\left(n_{e}(1), 0\right), \\
& p^{f}\left(n_{e}(1)-2,1\right)=\widetilde{A}+\widetilde{B}\left(\frac{\lambda(\lambda+\theta)}{\theta \mu}\right)^{n_{e}(1)-2}=\frac{\theta^{2} \mu \gamma}{\lambda^{2} q(\lambda+\theta)^{2}} p\left(n_{e}(1), 0\right),
\end{aligned}
$$

we solved that $\widetilde{A}=0$ and this followed that $p^{f}(n, 1)=\widetilde{B}\left(\frac{\lambda(\lambda+\theta)}{\theta \mu}\right)^{n}, \quad n=n_{e}(0)+1, \ldots, n_{e}(1)$. Then, by using (3.17) and (3.22), we can denote $p^{f}(n, i),(i=0,1,2)$ as

$$
\begin{array}{rlrl}
p^{f}(n, 1) & =\left(\frac{\theta \mu}{\lambda(\lambda+\theta)}\right)^{n_{e}(0)-n+1} p^{f}\left(n_{e}(0)+1,1\right), & & n=n_{e}(0)+1, \ldots, n_{e}(1), \\
p^{f}(n, 0) & =\frac{q \mu}{\gamma}\left(\frac{\theta \mu}{\lambda(\lambda+\theta)}\right)^{n_{e}(0)-n+1} p^{f}\left(n_{e}(0)+1,1\right), & & n=n_{e}(0)+1, \ldots, n_{e}(1), \\
p^{f}(n, 2)=\frac{\mu}{\lambda+\theta}\left(\frac{\theta \mu}{\lambda(\lambda+\theta)}\right)^{n_{e}(0)-n+1} p^{f}\left(n_{e}(0)+1,1\right), & n & =n_{e}(0)+1, \ldots, n_{e}(1) .
\end{array}
$$

To solve $p^{f}\left(n_{e}(0)+1,1\right)$, setting $n=n_{e}(0)$ in (3.17), (3.22) and (3.19), then ordering $n=n_{e}(0)+1$ in (3.19), we have that

$$
\begin{aligned}
\gamma p^{f}\left(n_{e}(0), 0\right) & =\lambda p^{f}\left(n_{e}(0)-1,0\right)+q \mu p^{f}\left(n_{e}(0), 1\right), \\
(\lambda+\mu) p^{f}\left(n_{e}(0), 1\right) & =\lambda p^{f}\left(n_{e}(0)-1,1\right)+\lambda p^{f}\left(n_{e}(0), 2\right)+\theta p^{f}\left(n_{e}(0)+1,2\right), \\
(\lambda+\theta) p^{f}\left(n_{e}(0), 2\right) & =\gamma p^{f}\left(n_{e}(0), 0\right)+p \mu p^{f}\left(n_{e}(0), 1\right), \\
(\lambda+\mu) p^{f}\left(n_{e}(0)+1,1\right) & =\lambda p^{f}\left(n_{e}(0), 1\right)+\lambda p^{f}\left(n_{e}(0)+1,2\right)+\theta p^{f}\left(n_{e}(0)+2,2\right) .
\end{aligned}
$$

We denote $p^{f}\left(n_{e}(0)+1,1\right)=g$, then $p^{f}\left(n_{e}(0)+1,0\right)=\frac{q \mu}{\gamma} g$ and $p^{f}\left(n_{e}(0)+1,2\right)=\frac{\mu}{\lambda+\theta} g, p^{f}\left(n_{e}(0)+2,2\right)=\frac{\lambda}{\theta} g$. By solving (3.29)-(3.32), we obtain

$$
\begin{aligned}
p^{f}\left(n_{e}(0), 0\right) & =\frac{\lambda q(\lambda+\theta)}{\theta \gamma} p^{f}\left(n_{e}(0)-1,1\right)+\frac{\lambda \theta+\lambda^{2} q}{\theta \gamma} p^{f}\left(n_{e}(0)-1,0\right), \\
p^{f}\left(n_{e}(0), 1\right) & =\frac{\lambda(\lambda+\theta)}{\mu \theta} p^{f}\left(n_{e}(0)-1,1\right)+\frac{\lambda^{2}}{\mu \theta} p\left({ }^{f} n_{e}(0)-1,0\right), \\
p^{f}\left(n_{e}(0), 2\right) & =\frac{\lambda}{\theta} p^{f}\left(n_{e}(0)-1,1\right)+\frac{\lambda}{\theta} p^{f}\left(n_{e}(0)-1,0\right), \\
g & =\left(\frac{\lambda(\lambda+\theta)}{\mu \theta}\right)^{2} p^{f}\left(n_{e}(0)-1,1\right)+\frac{\lambda^{3}(\lambda+\theta)}{\mu^{2} \theta^{2}} p^{f}\left(n_{e}(0)-1,0\right) .
\end{aligned}
$$

Hence, the boundary probabilities $p^{f}\left(n_{e}(0), i\right), i=0,1,2$ are derived. By putting (3.5)-(3.7) in equations (3.26), (3.18) and (3.21), where

$$
\begin{array}{r}
\sum_{i=1}^{3} \frac{\lambda \widetilde{c}_{i}}{\rho_{i}}=0, \\
\sum_{i=1}^{3}\left\{\frac{\lambda\left[(\lambda+\gamma) \rho_{i}-\lambda\right]}{q \mu \rho_{i}^{2}}\right\} \widetilde{c}_{i}=0, \\
\sum_{i=1}^{3}\left\{\frac{(\lambda+\gamma) \rho_{i}-\lambda}{q \mu \rho_{i}}\left[\frac{\lambda(\lambda+\mu) \rho_{i}-\lambda^{2}}{\left(\lambda+\theta \rho_{i}\right) \rho_{i}}-p \mu\right]-\gamma\right\} \widetilde{c}_{i}=0 .
\end{array}
$$




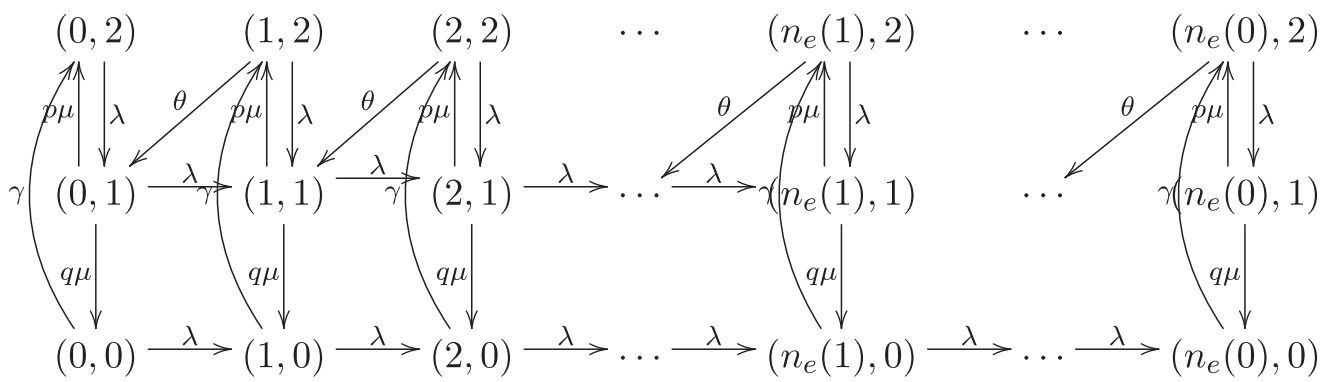

FIgURE 3. State transition diagram of the fully observable case when $n_{e}(0)>n_{e}(1)$.

TABLE 1. Steady-probabilities $p^{f}(n, 1)$ for $\mu=2.1, p=0.6, \gamma=1.5, \lambda=1, \theta=1.5, n_{e}(0)=$ $7, n_{e}(0)=8$.

\begin{tabular}{llllllllll}
\hline \hline$(i, n)$ & 0 & 1 & 2 & 3 & 4 & 5 & 6 & $n_{e}(0)$ & $n_{e}(1)$ \\
\hline$i=0$ & 0.0050 & 0.0119 & 0.0196 & 0.0275 & 0.0354 & 0.0433 & 0.0511 & 0.0773 & 0.0227 \\
$i=1$ & 0.0169 & 0.0299 & 0.0412 & 0.0519 & 0.0622 & 0.0723 & 0.0823 & 0.0528 & 0.0293 \\
$i=2$ & 0.0115 & 0.0172 & 0.0232 & 0.0293 & 0.0354 & 0.0414 & 0.0474 & 0.0444 & 0.0176 \\
\hline
\end{tabular}

TABLE 2. Steady-probabilities $p^{f}(n, 1)$ for $\mu=2.1, p=0.6, \gamma=1.5, \lambda=1, \theta=1.5, n_{e}(1)=$ $12, n_{e}(0)=13$.

\begin{tabular}{|c|c|c|c|c|c|c|c|c|c|c|c|c|c|c|}
\hline$(i, n)$ & 0 & 1 & 2 & 3 & 4 & 5 & 6 & 7 & 8 & 9 & 10 & 11 & $n_{e}(1)$ & $n_{e}(0)$ \\
\hline & & & & & & & & & & & & & 31 & 37 \\
\hline$i=1$ & 0.00 & 0.0 & 0 & & & 0 & 72 & 56 & & & 0 . & 0. & 0 & 089 \\
\hline$i=2$ & 0.0018 & 0.0033 & 0.0055 & 0.0083 & 0.0118 & 0.0161 & 0.0215 & 0.0282 & 0.0366 & 0.0470 & 0.0600 & 0.0644 & 0.0311 & 0.0187 \\
\hline
\end{tabular}

The constants $\widetilde{c}_{i}$ should be solved along with any two equations among (3.33)-(3.35) and the normalization condition $\sum_{i=0}^{2} \sum_{n=0}^{n_{e}(1)} p^{f}(n, i)=1$. Finally, we summarize all the probabilities in (3.5)-(3.13). We also give some numerical results in tabular below with the state probabilities obtained in Proposition 3.3, (see Tab. 1).

In what follows, the case when $n_{e}(0)>n_{e}(1)$ was considered and the stationary probabilities was summarized in the following (see Tab. 2).

Proposition 3.4. In the fully observable $M / M / 1$ constant retrial queue with Bernoulli vacations, when $n_{e}(0)>$ $n_{e}(1)$, the stationary distribution $\left(p^{f}(n, i):(n, i) \in\left\{0,1,2, \ldots, n_{e}\right\} \times\{0,1,2\}\right)$ are given as

$$
\begin{aligned}
p^{f}(n, 0) & =\widehat{c}_{1} \rho_{1}^{n}+\widehat{c}_{2} \rho_{2}^{n}+\widehat{c}_{3} \rho_{3}^{n}, & & n=0,1, \ldots, n_{e}(1)-1, \\
p^{f}(n, 1) & =\widehat{\nu}_{1} \rho_{1}^{n}+\widehat{\nu}_{2} \rho_{2}^{n}+\widehat{\nu}_{3} \rho_{3}^{n}, & & n=0,1, \ldots, n_{e}(1)-1, \\
p^{f}(n, 2) & =\widehat{\omega}_{1} \rho_{1}^{n}+\widehat{\omega}_{2} \rho_{2}^{n}+\widehat{\omega}_{3} \rho_{3}^{n}, & & \\
p^{f}\left(n_{e}(1), 0\right) & =\frac{\lambda(\lambda+\theta-\lambda p)}{\theta(\lambda p+\gamma)} \widehat{X}, & & \\
p^{f}\left(n_{e}(1), 1\right) & =\frac{\lambda^{2}(\lambda+\theta+\gamma)}{\mu \theta(\lambda p+\gamma)} \widehat{X}, & &
\end{aligned}
$$




$$
\begin{array}{rlrl}
p^{f}\left(n_{e}(1), 2\right) & =\frac{\lambda}{\theta} \widehat{X}, & \\
p^{f}(n, 0) & =\left(\frac{\theta(\lambda p+\gamma)}{\lambda(\lambda+\theta-\lambda p)}\right)^{n_{e}(1)-n-1} \widehat{X}, & & n=n_{e}(1)+1, \ldots, n_{e}(0)-1 \\
p^{f}(n, 1) & =\frac{\lambda(\lambda+\theta+\gamma)}{\mu(\lambda+\theta-\lambda p)}\left(\frac{\theta(\lambda p+\gamma)}{\lambda(\lambda+\theta-\lambda p)}\right)^{n_{e}(1)-n-1} \widehat{X}, & & n=n_{e}(1)+1, \ldots, n_{e}(0)-1, \\
p^{f}(n, 2) & =\frac{\lambda p+\gamma}{\lambda+\theta-\lambda p}\left(\frac{\theta(\lambda p+\gamma)}{\lambda(\lambda+\theta-\lambda p)}\right)^{n_{e}(1)-n-1} \widehat{X}, & & n=n_{e}(1)+1, \ldots, n_{e}(0)-1, \\
p^{f}\left(n_{e}(0), 0\right) & =\frac{\lambda(\lambda+\theta-\lambda p)}{\theta \gamma}\left(\frac{\theta(\lambda p+\gamma)}{\lambda(\lambda+\theta-\lambda p)}\right)^{n_{e}(1)-n_{e}(0)} \widehat{X}, & \\
p^{f}\left(n_{e}(0), 1\right) & =\frac{\lambda^{2}}{\theta \mu}\left(\frac{\theta(\lambda p+\gamma)}{\lambda(\lambda+\theta-\lambda p)}\right)^{n_{e}(1)-n_{e}(0)} \widehat{X}, & \\
p^{f}\left(n_{e}(0), 2\right) & =\frac{\lambda}{\theta}\left(\frac{\theta(\lambda p+\gamma)}{\lambda(\lambda+\theta-\lambda p)}\right)^{n_{e}(1)-n_{e}(0)} \widehat{X},
\end{array}
$$

where

$$
\begin{array}{rlr}
\widehat{\nu}_{i} & =\frac{(\lambda+\gamma) \rho_{i}-\lambda}{q \mu \rho_{i}} \widehat{c}_{i}, & i=1,2,3 \\
\widehat{\omega}_{i} & =\frac{(\lambda+\mu) \rho_{i}-\lambda}{\left(\lambda+\theta \rho_{i}\right) \rho_{i}} \widehat{\nu}_{i}, & i=1,2,3 \\
a & =-\frac{(\lambda+\mu)(\lambda+\gamma)(\lambda+\theta)-\lambda \mu(\lambda p+\gamma)+\lambda p \theta \mu}{\mu \theta(\lambda p+\gamma)}, & \\
b & =\frac{\lambda\{(\lambda+\theta)(2 \lambda+\mu+\gamma)-\lambda p \mu\}}{\mu \theta(\lambda p+\gamma)}, \\
c & =-\frac{\lambda^{2}(\lambda+\theta)}{\mu \theta(\lambda p+\gamma)}, \\
\widehat{X} & =\widehat{c}_{1} \rho_{1}^{n_{e}(1)-1}+\widehat{c}_{2} \rho_{2}^{n_{e}(1)-1}+\widehat{c}_{3} \rho_{3}^{n_{e}(1)-1} .
\end{array}
$$

The coefficient $\widehat{c}_{i}(i=1,2,3)$ were solved by using normalization condition and $\rho_{i}(i=1,2,3)$ are three roots of equation $x^{3}+a x^{2}+b x+c=0$.

Proof. In this situation, the state transition diagram is shown in Figure 3, and the balance equations are given as

$$
\begin{array}{rlrl}
(\lambda+\gamma) p^{f}(0,0) & =q \mu p^{f}(0,1), & & \\
(\lambda+\gamma) p^{f}(n, 0) & =\lambda p^{f}(n-1,0)+q \mu p^{f}(n, 1), & n=1,2, \ldots, n_{e}(0)-1, \\
\gamma p^{f}\left(n_{e}(0), 0\right) & =\lambda p^{f}\left(n_{e}(0)-1,0\right)+q \mu p^{f}\left(n_{e}(0), 1\right), & & \\
(\lambda+\mu) p^{f}(0,1) & =\lambda p^{f}(0,2)+\theta p^{f}(1,2), & n=1,2, \ldots, n_{e}(1)-1, \\
(\lambda+\mu) p^{f}(n, 1) & =\lambda p^{f}(n-1,1)+\lambda p^{f}(n, 2)+\theta p^{f}(n+1,2), & & \\
\mu p^{f}\left(n_{e}(1), 1\right) & =\lambda p^{f}\left(n_{e}(1)-1,1\right)+\lambda p^{f}\left(n_{e}(1), 2\right)+\theta p^{f}\left(n_{e}(1)+1,2\right), & & \\
\mu p^{f}(n, 1) & =\lambda p^{f}(n, 2)+\theta p^{f}(n+1,2), & & \\
\mu p^{f}\left(n_{e}(0), 1\right) & =\lambda p^{f}\left(n_{e}(0), 2\right), & & \\
\lambda p^{f}(0,2) & =\gamma p^{f}(0,0)+p \mu p^{f}(0,1), & n=1,2, \ldots, n_{e}(0) . & \\
(\lambda+\theta) p^{f}(n, 2) & =\gamma p^{f}(n, 0)+p \mu p^{f}(n, 1), &
\end{array}
$$


Also, the stationary distribution is the unique positive solution of the balance equations above. Similarly, combining with (3.49), (3.52) and (3.57), we obtain

$$
\begin{aligned}
& -\mu \theta(\lambda p+\gamma) p^{f}(n+1,0)+\{(\lambda+\mu)(\lambda+\gamma)(\lambda+\theta)-\lambda \mu(\lambda p+\gamma)+\lambda p \theta \mu\} p^{f}(n, 0) \\
& -\lambda\{(\lambda+\theta)(2 \lambda+\mu+\gamma)-\lambda p \mu\} p^{f}(n-1,0)+\lambda^{2}(\lambda+\theta) p^{f}(n-2,0)=0, n=2, \ldots, n_{e}(1)-2,
\end{aligned}
$$

which is a three-order difference equation with solutions $\rho_{i}(i=1,2,3)$. Therefore, we can set

$$
p^{f}(n, 0)=\widehat{c}_{1} \rho_{1}^{n}+\widehat{c}_{2} \rho_{2}^{n}+\widehat{c}_{3} \rho_{3}^{n}, \quad n=0,1, \ldots, n_{e}(1)-1,
$$

where $\widehat{c}_{i}(i=1,2,3)$ are undetermined constants we are going to explore next. Similar to method used in Proposition 3.3, the form of $p^{f}(n, i),\left(i=0,1,2, n=0,1, \ldots, n_{e}(1)-1\right)$ are concluded in (3.36)-(3.38). Then, combining (3.49), (3.54) and (3.57), we have

$$
\begin{aligned}
\theta(\lambda p+\gamma) p^{f}(n+1,0) & -((\lambda+\theta)(\lambda+\gamma)-\lambda(\lambda p+\gamma)+\lambda \theta p) p^{f}(n, 0)+\lambda(\lambda q+\theta) p^{f}(n-1,0)=0, \\
n & =n_{e}(1)+1, \ldots, n_{e}(0)-1,
\end{aligned}
$$

which can be considered as a homogeneous linear difference equation and the characteristic equation is

$$
\theta(\lambda p+\gamma) x^{2}-((\lambda+\theta)(\lambda+\gamma)-\lambda(\lambda p+\gamma)+\lambda \theta p) x+\lambda(\lambda q+\theta)=0, \quad n=n_{e}(1)+1, \ldots, n_{e}(0)-1
$$

It has two roots, 1 and $\frac{\lambda(\lambda+\theta)-\lambda^{2} p}{\theta(\lambda p+\gamma)}$, where the probability $p^{f}(n, 0)$ can be denoted by

$$
p^{f}(n, 0)=\widehat{A} 1^{n}+\widehat{B}\left(\frac{\lambda(\lambda+\theta)-\lambda^{2} p}{\theta(\lambda p+\gamma)}\right)^{n}, \quad n=n_{e}(0)+1, \ldots, n_{e}(1)-1,
$$

and $\widehat{A}, \widehat{B}$ in (3.59) are undetermined constants that we have to solve. By using similar computing method in Proposition 3.3 , we also have that $\widehat{A}=0$ and then

$$
p^{f}(n, 0)=\widehat{B}\left(\frac{\lambda(\lambda+\theta)-\lambda^{2} p}{\theta(\lambda p+\gamma)}\right)^{n}, \quad n=n_{e}(1)+1, \ldots, n_{e}(0)-1 .
$$

By using (3.26) and (3.31), we can denote $p^{f}(n, i),(i=0,1,2)$ as

$$
\begin{array}{ll}
p^{f}(n, 0)=\left(\frac{\theta(\lambda p+\gamma)}{\lambda(\lambda+\theta)-\lambda^{2} p}\right)^{n_{e}(1)-n+1} p^{f}\left(n_{e}(1)+1,0\right), & n=n_{e}(1)+1, \ldots, n_{e}(0)-1, \\
p^{f}(n, 1)=\frac{\lambda(\lambda+\theta+\gamma)}{\mu(\lambda+\theta-\lambda p)}\left(\frac{\theta(\lambda p+\gamma)}{\lambda(\lambda+\theta)-\lambda^{2} p}\right)^{n_{e}(1)-n+1} p^{f}\left(n_{e}(1)+1,0\right), & n=n_{e}(1)+1, \ldots, n_{e}(0)-1, \\
p^{f}(n, 2)=\frac{\lambda p+\gamma}{\lambda+\theta-\lambda p}\left(\frac{\theta(\lambda p+\gamma)}{\lambda(\lambda+\theta)-\lambda^{2} p}\right)^{n_{e}(1)-n+1} p^{f}\left(n_{e}(1)+1,0\right), & n=n_{e}(1)+1, \ldots, n_{e}(0)-1 .
\end{array}
$$

By ordering $n=n_{e}(1)$ in (3.49), (3.54) and (3.57), and order $n=n_{e}(1)+1$ in (3.49), we have that

$$
\begin{aligned}
(\lambda+\gamma) p^{f}\left(n_{e}(1), 0\right) & =\lambda p^{f}\left(n_{e}(1)-1,0\right)+q \mu p^{f}\left(n_{e}(1), 1\right), \\
(\lambda+\gamma) p^{f}\left(n_{e}(1)+1,0\right) & =\lambda p^{f}\left(n_{e}(1), 0\right)+q \mu p^{f}\left(n_{e}(1)+1,1\right), \\
\mu p^{f}\left(n_{e}(1), 1\right) & =\lambda p^{f}\left(n_{e}(1), 2\right)+\theta p^{f}\left(n_{e}(1)+1,2\right), \\
(\lambda+\theta) p^{f}\left(n_{e}(1), 2\right) & =\gamma p^{f}\left(n_{e}(1), 0\right)+p \mu p^{f}\left(n_{e}(1), 1\right) .
\end{aligned}
$$


We denote $p^{f}\left(n_{e}(1)+1,1\right)=h$ and solved that

$$
\begin{aligned}
p^{f}\left(n_{e}(1), 0\right) & =\frac{\lambda(\lambda+\theta-\lambda p)}{\theta(\lambda p+\gamma)} p^{f}\left(n_{e}(1)-1,0\right), \\
p^{f}\left(n_{e}(1), 1\right) & =\frac{\lambda^{2}(\lambda+\theta+\gamma)}{\mu \theta(\lambda p+\gamma)} p^{f}\left(n_{e}(1)-1,0\right), \\
p^{f}\left(n_{e}(1), 2\right) & =\frac{\lambda}{\theta} p^{f}\left(n_{e}(1)-1,0\right), \\
h & =\frac{\lambda^{3}(\lambda+\theta-\lambda p)(\lambda+\theta+\gamma)}{\mu \theta^{2}(\lambda p+\gamma)^{2}} p^{f}\left(n_{e}(1)-1,0\right) .
\end{aligned}
$$

Finally, the probability $p^{f}\left(n_{e}(0), i\right),(i=0,1,2)$ were solved by considering (3.50), (3.55) and (3.57). By putting (3.36)-(3.38) in equations (3.48), (3.51) and (3.56), where

$$
\begin{array}{r}
\sum_{i=1}^{3} \frac{\lambda \widehat{c}_{i}}{\rho_{i}}=0, \\
\sum_{i=1}^{3}\left\{\frac{\lambda\left[(\lambda+\gamma) \rho_{i}-\lambda\right]}{q \mu \rho_{i}^{2}}\right\} \widehat{c}_{i}=0, \\
\sum_{i=1}^{3}\left\{\frac{(\lambda+\gamma) \rho_{i}-\lambda}{q \mu \rho_{i}}\left[\frac{\lambda(\lambda+\mu) \rho_{i}-\lambda^{2}}{\left(\lambda+\theta \rho_{i}\right) \rho_{i}}-p \mu\right]-\gamma\right\} \widehat{c}_{i}=0 .
\end{array}
$$

The constants $\widehat{c}_{i}$ should be solved along with any two equations among (3.59)-(3.61) and the normalization condition $\sum_{i=0}^{2} \sum_{n=0}^{n_{e}(0)} p^{f}(n, i)=1$. Then we summarize all the probabilities in (3.36)-(3.47). We also give some numerical results in tabular below with the state probabilities obtained in Proposition 3.4.

- When $n_{e}(0)=n_{e}(1)$, the state transition diagram of this case is the same as the almost observable case we are going to discuss next, thus we omit the calculation process of the stationary distribution here.

Since the balking probabilities are $p^{f}\left(n_{e}(1), 1\right)+\sum_{n=n_{e}(0)}^{n_{e}(1)} p^{f}(n, 0)$ and $p^{f}\left(n_{e}(0), 0\right)+\sum_{n=n_{e}(1)}^{n_{e}(0)} p^{f}(n, 1)$ in the first two cases respectively, the corresponding social benefits when all follow the pair of thresholds $\left(n_{e}(0), n_{e}(1)\right)$ are given as

- If $n_{e}(0)<n_{e}(1)$

$$
S_{\mathrm{soc}}^{f}=\lambda R\left(1-p^{f}\left(n_{e}(1), 1\right)-\sum_{n=n_{e}(0)}^{n_{e}(1)} p^{f}(n, 0)\right)-C \sum_{n=0}^{n_{e}(1)} n\left(p^{f}(n, 0)+p^{f}(n, 1)+p^{f}(n, 2)\right),
$$

where $p^{f}(n, i)(i=0,1,2)$ in (3.62) are given by (3.26)-(3.22);

- If $n_{e}(0)>n_{e}(1)$

$$
S_{\mathrm{soc}}^{f}=\lambda R\left(1-p^{f}\left(n_{e}(0), 0\right)-\sum_{n=n_{e}(1)}^{n_{e}(0)} p^{f}(n, 1)\right)-C \sum_{n=0}^{n_{e}(0)} n\left(p^{f}(n, 0)+p^{f}(n, 1)+p^{f}(n, 2)\right),
$$

where $p^{f}(n, i)(i=0,1,2)$ in (3.63) are given by (3.48)-(3.57); 
- If $n_{e}(0)=n_{e}(1)=n^{*}$

$$
S_{\text {soc }}^{f}=\lambda R\left(1-p^{f}\left(n^{*}, 1\right)-p^{f}\left(n^{*}, 0\right)\right)-C \sum_{n=0}^{n^{*}} n\left(p^{f}(n, 0)+p^{f}(n, 1)+p^{f}(n, 2)\right),
$$

where $p^{f}(n, i)(i=0,1,2)$ in (3.64) can be solved in the almost observable case.

\subsection{Almost observable queue}

Now we turn to the case where blocked customers can only observe the number of customers in the orbit upon arrivals. The state transition diagram is depicted in Figure 4. We explore the corresponding equilibrium strategy and investigate that it is a pure threshold strategy. Therefore, it is necessary to solve the stationary distribution in this system when all blocked ones follow the given threshold strategy. The results are summarized in the following (see Tab. 2).

Theorem 3.5. In the almost observable $M / M / 1$ constant retrial queue with Bernoulli vacations, where all blocked customers follow the strategy "joining the system if $N(t) \leq n_{e}-1$ and balking otherwise", the stationary distribution $\left(p^{a}(n, i):(n, i) \in\left\{0,1,2, \ldots, n_{e}\right\} \times\{0,1,2\}\right)$ are given as

$$
\begin{array}{rlrl}
p^{a}(n, 0) & =c_{1} \rho_{1}^{n}+c_{2} \rho_{2}^{n}+c_{3} \rho_{3}^{n}, & n=0,1, \ldots, n_{e}-1, \\
p^{a}(n, 1) & =\nu_{1} \rho_{1}^{n}+\nu_{2} \rho_{2}^{n}+\nu_{3} \rho_{3}^{n}, & n=0,1, \ldots, n_{e}-1, \\
p^{a}(n, 2) & =\omega_{1} \rho_{1}^{n}+\omega_{2} \rho_{2}^{n}+\omega_{3} \rho_{3}^{n}, & & n=0,1, \ldots, n_{e}-1, \\
p^{a}\left(n_{e}, 0\right) & =\frac{\lambda}{\gamma}\left[\left(1+\frac{\lambda q}{\theta}\right) \sum_{i=1}^{3} c_{i} \rho_{i}^{n_{e}-1}+\left(q+\frac{\lambda q}{\theta}\right) \sum_{i=1}^{3} \nu_{i} \rho^{n_{e}-1}\right], & \\
p^{a}\left(n_{e}, 1\right) & =\frac{\lambda}{\theta \mu}\left[\lambda \sum_{i=1}^{3} c_{i} \rho_{i}^{n_{e}-1}+(\lambda+\theta) \sum_{i=1}^{3} \nu_{i} \rho_{i}^{n_{e}-1}\right], \\
p^{a}\left(n_{e}, 2\right) & =\frac{\lambda}{\theta}\left[\sum_{i=1}^{3} c_{i} \rho_{i}^{n_{e}-1}+\sum_{i=1}^{3} \nu_{i} \rho_{i}^{n_{e}-1}\right], & &
\end{array}
$$

where

$$
\begin{array}{rlr}
\nu_{i} & =\frac{(\lambda+\gamma) \rho_{i}-\lambda}{q \mu \rho_{i}} c_{i}, & i=1,2,3 \\
\omega_{i} & =\frac{(\lambda+\mu) \rho_{i}-\lambda}{\left(\lambda+\theta \rho_{i}\right) \rho_{i}} \nu_{i}, & i=1,2,3 \\
a & =-\frac{(\lambda+\mu)(\lambda+\gamma)(\lambda+\theta)-\lambda \mu(\lambda p+\gamma)+\lambda p \theta \mu}{\mu \theta(\lambda p+\gamma)}, & \\
b & =\frac{\lambda\{(\lambda+\theta)(2 \lambda+\mu+\gamma)-\lambda p \mu\}}{\mu \theta(\lambda p+\gamma)}, & \\
c & =-\frac{\lambda^{2}(\lambda+\theta)}{\mu \theta(\lambda p+\gamma)}
\end{array}
$$

The coefficient $c_{i}(i=1,2,3)$ were solved by using normalization condition and $\rho_{i}(i=1,2,3)$ are three roots of equation $x^{3}+a x^{2}+b x+c=0$.

Proof. According to Figure 4, the stationary distribution $\left(p(n, i):(n, i) \in\left\{0,1,2, \ldots, n_{e}\right\} \times\{0,1,2\}\right)$ were determined by balance equations:

$$
(\lambda+\gamma) p^{a}(0,0)=q \mu p^{a}(0,1),
$$




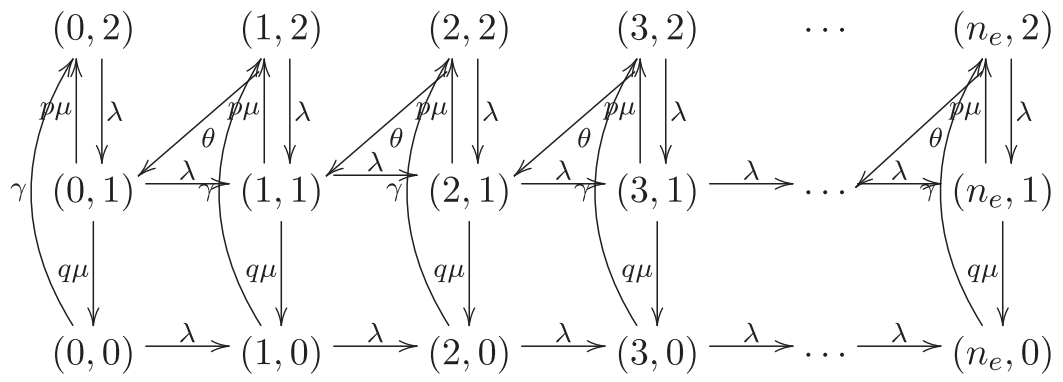

FiguRE 4. Transition rate diagram of the almost observable case.

$$
\begin{array}{rlrl}
(\lambda+\gamma) p^{a}(n, 0) & =\lambda p^{a}(n-1,0)+q \mu p^{a}(n, 1), & & n=1,2, \ldots, n_{e}-1, \\
\gamma p^{a}\left(n_{e}, 0\right) & =\lambda p^{a}\left(n_{e}-1,0\right)+q \mu p^{a}\left(n_{e}, 1\right), & & \\
(\lambda+\mu) p^{a}(0,1) & =\lambda p^{a}(0,2)+\theta p^{a}(1,2), & & \\
(\lambda+\mu) p^{a}(n, 1) & =\lambda p^{a}(n-1,1)+\lambda p^{a}(n, 2)+\theta p^{a}(n+1,2), & & \\
\mu p^{a}\left(n_{e}, 1\right) & =\lambda p^{a}\left(n_{e}-1,1\right)+\lambda p^{a}\left(n_{e}, 2\right), n_{e}-1, \\
\lambda p^{a}(0,2) & =\gamma p^{a}(0,0)+p \mu p^{a}(0,1), & & \\
(\lambda+\theta) p^{a}(n, 2) & =\gamma p^{a}(n, 0)+p \mu p^{a}(n, 1), & & n=1,2, \ldots, n_{e} .
\end{array}
$$

Combining with equations (3.72), (3.75) and (3.78), we get:

$$
\begin{aligned}
& -\mu \theta(\lambda p+\gamma) p^{a}(n+1,0)+\{(\lambda+\mu)(\lambda+\gamma)(\lambda+\theta)-\lambda \mu(\lambda p+\gamma)+\lambda p \theta \mu\} p^{a}(n, 0) \\
& -\lambda\{(\lambda+\theta)(2 \lambda+\mu+\gamma)-\lambda p \mu\} p^{a}(n-1,0)+\lambda^{2}(\lambda+\theta) p^{a}(n-2,0)=0, \quad n=2, \ldots, n_{e}-2,
\end{aligned}
$$

which is a three-order difference equation with solutions $\rho_{i}(i=1,2,3)$. Therefore, we can set the stationary probability of $I(t)=0$ as

$$
p^{a}(n, 0)=c_{1} \rho_{1}^{n}+c_{2} \rho_{2}^{n}+c_{3} \rho_{3}^{n}, \quad n=0,1, \ldots, n_{e}-1,
$$

where $c_{i}(i=1,2,3)$ are undetermined constants we are going to explore next. By plugging (3.79) in (3.72), we get

$$
p^{a}(n, 1)=\nu_{1} \rho_{1}^{n}+\nu_{2} \rho_{2}^{n}+\nu_{3} \rho_{3}^{n}, \quad n=1,2, \ldots, n_{e}-1 .
$$

Again, by plugging equations (3.79) and (3.80) in (3.78), we have

$$
p^{a}(n, 2)=\omega_{1} \rho_{1}^{n}+\omega_{2} \rho_{2}^{n}+\omega_{3} \rho_{3}^{n}, \quad n=1,2, \ldots, n_{e}-1 .
$$

By ordering $n=1$ in (3.72) and using (3.80) and (3.81), it is followed that:

$$
p^{a}(0,1)=\nu_{1}+\nu_{2}+\nu_{3} .
$$

Then the general formula of $p^{a}(n, 1)$ in (3.66) is obtained by combining with (3.80) and the above equation. Similarly, when ordering $n=1$ in (3.78), we get (3.67) with the help of equations (3.79) and (3.81). The results (3.68)-(3.70) are derived by using (3.65)-(3.67) based on equations (3.73), (3.76) and (3.78). In addition, the following equations were calculated by putting (3.65)-(3.67) in equations (3.71), (3.74) and (3.77), where 
TABLE 3. Steady-probabilities for $\mu=1.2, p=0.8, \gamma=1.5, \lambda=0.5, \theta=1.5, n_{e}=11$.

\begin{tabular}{llllllllllll}
\hline \hline$p^{a}(n, i)$ & 0 & 1 & 2 & 4 & 5 & 6 & 7 & 8 & 9 & 10 & 11 \\
\hline$i=0$ & 0.0028 & 0.0052 & 0.0069 & 0.0080 & 0.0088 & 0.0092 & 0.0095 & 0.0097 & 0.0098 & 0.0099 & 0.0099 \\
$i=1$ & 0.0234 & 0.0278 & 0.0368 & 0.0473 & 0.0562 & 0.0585 & 0.0599 & 0.0609 & 0.0615 & 0.0618 & 0.0621 \\
$i=2$ & 0.0193 & 0.0234 & 0.0280 & 0.0313 & 0.0335 & 0.0350 & 0.0359 & 0.0365 & 0.0369 & 0.0371 & 0.0372 \\
\hline
\end{tabular}

$$
\begin{array}{r}
\sum_{i=1}^{3} \frac{\lambda c_{i}}{\rho_{i}}=0, \\
\sum_{i=1}^{3}\left\{\frac{\lambda\left[(\lambda+\gamma) \rho_{i}-\lambda\right]}{q \mu \rho_{i}^{2}}\right\} c_{i}=0, \\
\sum_{i=1}^{3}\left\{\frac{(\lambda+\gamma) \rho_{i}-\lambda}{q \mu \rho_{i}}\left[\frac{\lambda(\lambda+\mu) \rho_{i}-\lambda^{2}}{\left(\lambda+\theta \rho_{i}\right) \rho_{i}}-p \mu\right]-\gamma\right\} c_{i}=0 .
\end{array}
$$

The constants $c_{i}(i=1,2,3)$ should be solved along with any two equations among (3.83)-(3.85) and the normalization condition $\sum_{i=0}^{2} \sum_{n=0}^{n_{e}} p^{a}(n, i)=1$. Some numerical results in tabular below with the state probabilities of this case are shown in Table 3 .

We then proceed to the expected utility of a joining customer when he observes $n$ customers ahead of him in the orbit.

Proposition 3.6. In the almost observable $M / M / 1$ constant retrial queue with Bernoulli vacations, blocked customers all follow the threshold strategy "join the system if $N(t) \leq n_{e}-1$ and balks otherwise at time $t$ ". The utility of a joining customer when he observes $n$ customers in the orbit is given by

$$
\begin{aligned}
S(n) & =R-C\left(\frac{[\mu \gamma+(q \mu+\gamma)(\lambda+\theta)](n+1)}{\mu \theta \gamma}+\frac{(p \mu-\gamma) \sum_{i=1}^{3} c_{i} \rho_{i}^{n}}{\mu \gamma \sum_{i=1}^{3}\left(c_{i}+\nu_{i}\right) \rho_{i}^{n}}+\frac{1}{\mu}\right), \quad n=0, \ldots, n_{e}-1, \\
S\left(n_{e}\right) & =R-C\left(\frac{[\mu \gamma+(q \mu+\gamma)(\lambda+\theta)]\left(n_{e}+1\right)}{\mu \theta \gamma}+\frac{(p \mu-\gamma) p^{a}\left(n_{e}, 0\right)}{\mu \gamma\left(p^{a}\left(n_{e}, 0\right)+p^{a}\left(n_{e}, 1\right)\right)}+\frac{1}{\mu}\right),
\end{aligned}
$$

where $p^{a}\left(n_{e}, 0\right), p^{a}\left(n_{e}, 1\right)$ are given by Theorem 3.5.

Proof. The expected net benefit of a joining blocked customer when there are $n$ customers before him upon arrival is

$$
S(n)=R-C\left(T(n+1)+\frac{1}{\mu}\right)
$$

where $T(n+1)=E\left[S \mid N^{-}=n\right]$ is his mean sojourn time in the orbit given that he observes $n$ customers upon arrival. We denote $\operatorname{Pr}\left(I^{-}=i \mid N^{-}=n\right)$ as the conditional probability that the state is $i(i=0,1)$ if he observes $n$ customers in the orbit upon his arrival. Conditioning on the state of server upon his arrival and taking (3.2) and (3.3) into account, we obtain:

$$
\begin{aligned}
T(n+1) & =T(n+1,1) \operatorname{Pr}\left(I^{-}=1 \mid N^{-}=n\right)+T(n+1,0) \operatorname{Pr}\left(I^{-}=0 \mid N^{-}=n\right) \\
& =T(n+1,1)+\frac{p \mu-\gamma}{\mu \gamma} \times \operatorname{Pr}\left(I^{-}=0 \mid N^{-}=n\right),
\end{aligned}
$$


where $T(n+1,1)$ is given by $(3.3)$, and

$$
\operatorname{Pr}\left(I^{-}=0 \mid N^{-}=n\right)=\frac{p^{a}(n, 0)}{p^{a}(n, 0)+p^{a}(n, 1)}, \quad n=0,1, \ldots, n_{e} .
$$

Combining with the stationary probabilities obtained in Theorem 3.5, we get $\operatorname{Pr}\left(I^{-}=0 \mid N^{-}=n\right)(n=$ $\left.0,1, \ldots, n_{e}\right)$. Hence, we have

$$
\begin{aligned}
T(n+1) & =\frac{[\mu \gamma+(q \mu+\gamma)(\lambda+\theta)](n+1)}{\mu \theta \gamma}+\frac{(p \mu-\gamma) \sum_{i=1}^{3} c_{i} \rho_{i}^{n}}{\mu \gamma \sum_{i=1}^{3}\left(c_{i}+\nu_{i}\right) \rho_{i}^{n}}, \quad n=0,1, \ldots, n_{e}-1, \\
T\left(n_{e}+1\right) & =\frac{[\mu \gamma+(q \mu+\gamma)(\lambda+\theta)]\left(n_{e}+1\right)}{\mu \theta \gamma}+\frac{(p \mu-\gamma) p^{a}\left(n_{e}, 0\right)}{\mu \gamma\left(p^{a}\left(n_{e}, 0\right)+p^{a}\left(n_{e}, 1\right)\right)},
\end{aligned}
$$

where probabilities $p^{a}\left(n_{e}, 0\right), p^{a}\left(n_{e}, 1\right)$ and $p^{a}\left(n_{e}, 2\right)$ have been given in equations (3.68)-(3.70). Therefore, the corresponding expected benefit in equations (3.86) and (3.87) are given by plugging (3.89) and (3.90) in (3.88).

What's more, if $S(0)<0$, no blocked customer enters the system even if there is no customer in the orbit. So we might assume that $S(0)>0$, then the equilibrium threshold strategy in the almost observable case was summarized as follows.

Theorem 3.7. Define the functions $f_{1}(n)$ and $f_{2}(n)$ by

$$
\begin{aligned}
f_{1}(n) & =R-C\left\{\frac{[\mu \gamma+(q \mu+\gamma)(\lambda+\theta)](n+1)}{\mu \theta \gamma}+\frac{1}{\mu}+\frac{(p \mu-\gamma) \sum_{i=1}^{3} c_{i} \rho_{i}^{n}}{\mu \gamma \sum_{i=1}^{3}\left(c_{i}+\nu_{i}\right) \rho_{i}^{n}}\right\}, \quad n=0,1, \ldots, \\
f_{2}(n) & =R-C\left\{\frac{[\mu \gamma+(q \mu+\gamma)(\lambda+\theta)](n+1)}{\mu \theta \gamma}+\frac{1}{\mu}+\frac{(p \mu-\gamma)\left((\lambda q+\theta) \Phi_{1}+(\lambda+\theta) q \Phi_{2}\right)}{((\lambda q+\theta) \mu+\lambda \gamma) \gamma \Phi_{1}+(\lambda+\theta)(q \mu+\gamma) \gamma \Phi_{2}}\right\}, \\
n & =0,1, \ldots,
\end{aligned}
$$

where $\Phi_{1}=\sum_{i=1}^{3} c_{i} \rho_{i}^{n-1}$ and $\Phi_{2}=\sum_{i=1}^{3} \nu_{i} \rho_{i}^{n-1}$.

By definition, we have that $f_{1}(n)=S(n),\left(n=0,1, \ldots, n_{e}-1\right)$ and $f_{2}\left(n_{e}\right)=S\left(n_{e}\right)$. In addition, $S(n)$ is decreasing in $n$ and this indicates that $f_{1}(n)>f_{2}(n)$, where $n=0,1, \cdots$.

Then there exists non-negative integers upper bound $n_{U}$ and lower bound $n_{L}\left(n_{L} \leq n_{U}\right)$ which satisfy

$$
f_{1}(0)>f_{1}(1)>\cdots>f_{1}\left(n_{U}-1\right)>0, \quad f_{1}\left(n_{U}\right) \leq 0,
$$

and

$$
f_{2}\left(n_{L}-1\right)>0, \quad f_{2}\left(n_{U}\right)<f_{2}\left(n_{U}-1\right)<\cdots<f_{2}\left(n_{L}+1\right)<f_{2}\left(n_{L}\right) \leq 0,
$$

or

$$
0 \geq f_{2}(0)>\cdots>f_{2}\left(n_{U}-2\right)>f_{2}\left(n_{U}-1\right)>f_{2}\left(n_{U}\right) .
$$

In the almost observable $M / M / 1$ constant retrial queue with Bernoulli vacations, blocked customers follow the pure threshold strategy "join if $N(t) \leq n_{e}-1$ and balks otherwise at time $t$ ". Moreover, the equilibrium threshold $n_{e} \in\left\{n_{L}, n_{L}+1, \ldots, n_{U}\right\}$. 
Proof. Since we have assumed the basic condition $S(0)>0$ and $f_{1}(n)$ decreases in $n, f_{1}(0)>0$ and $\lim _{n \rightarrow \infty} f_{1}(n)=$ $-\infty$ are founded. If $n_{U}$ is the first subscript of the sequence of non-positive values of $\left\{f_{1}(n), n=0,1, \ldots\right\}$, the condition (3.93) holds. Recall that $f_{1}(n)>f_{2}(n),(n=0,1, \ldots)$, thus the inequality $f_{2}\left(n_{U}\right)<f_{1}\left(n_{U}\right) \leq 0$ is established. Starting from the subscript $n_{U}$ then towards to 0 , and we let $f_{2}\left(n_{L}-1\right)$ be the first positive term of $f_{2}(n)$, that is, the condition (3.94) holds. Otherwise, the values of $f_{2}(n),\left(n=0,1, \ldots, n_{U}\right)$ are all non-positive, and this is equivalent to $(3.95)$.

Now the existence of equilibrium threshold strategy is found in the almost observable case. We consider a tagged customer while all others follow the same threshold strategy "join the system if $N(t) \leq n_{e}-1$ and balks otherwise at time $t$ when find unavailable server" for the fixed $n_{e} \in\left\{n_{L}, n_{L}+1, \ldots, n_{U}\right\}$.

- If the tagged one observes $n\left(\leq n_{e}-1\right)$ customers ahead of him in the orbit, the expected benefit of joining is $f_{1}(n)>0$ and he prefers to join the system.

- If he observes $n_{e}$ customers in the orbit and decides to join, the expected benefit of him is $f_{2}\left(n_{e}\right) \leq 0$ by equations (3.88), (3.92), (3.94) and (3.95), so in this case he prefers to balk.

The social benefits per unit time when all blocked customers follow the pure threshold strategy $n_{e}$ is

$$
S_{\mathrm{soc}}^{a}=\lambda R\left(1-p^{a}\left(n_{e}, 1\right)-p^{a}\left(n_{e}, 0\right)\right)-C \sum_{n=0}^{n_{e}} n p^{a}(n),
$$

where

$$
p^{a}(n)=p^{a}(n, 1)+p^{a}(n, 0)+p^{a}(n, 2), \quad n=1,2, \ldots, n_{e},
$$

and $p^{a}(n, i)$ are determined by Theorem 3.5.

Remark 3.8. The results in Theorem 3.7 provide a searching method to identify the equilibrium strategy in the almost observable case. Firstly, starting from 0 to search for the first negative term in $\left\{f_{1}(n), 0,1, \ldots\right\}$, then denote the subscript of it as $n_{U}$ (upper equilibrium threshold). Besides, we compute $f_{2}(n)$ from $n_{U}$ to 0 until finding the first positive term of the sequences, that is $n_{L}$ (lower equilibrium threshold).

Remark 3.9. We prove a "Follow the crowd" (FTC) situation in this system where the joining decision of an individual is increasing with the one adopted by the others in the almost observable case. In our model, we denote the expected joining benefit of a customer under threshold $n_{e}$ and observes $n$ customers before him in the orbit is $S_{n_{e}}(n)$. Now we assume that the others adopt the threshold $n_{e}+1$, the expected benefit $S_{n_{e}+1}(n)$ is the same as $S_{n_{e}}(n)$ for $n=0,1, \ldots, n_{e}-1$ but $S_{n_{e}+1}\left(n_{e}\right)>S_{n_{e}}\left(n_{e}\right)$. That is to say, a blocked customer is more likely to join if the others follow a higher threshold, i.e., he follows the others' behavior.

\section{NumERICAL EXAMPLES}

In this section, we explore the effects of several parameters on customers' equilibrium strategies in different information levels by numerical scenarios. We show the sensitivity of equilibrium thresholds with respect to the main parameters: $\theta, \gamma, \mu$ and $p$. Besides, it is obvious that these joining thresholds are increasing in service reward $R$ and decreasing in unit waiting cost $C$. Hence in all applications, we set $R=25$ and $C=1$.

Figures 5-8 show the variation tendency of $n_{e}(0), n_{e}(1)$ in the fully observable case. More concretely, in Figure 5, we observe that both the thresholds $n_{e}(0)$ and $n_{e}(1)$ are non decreasing functions of retrial rate $\theta$. This is because customers in the orbit are more likely to be searched by available server with a higher retrial rate. The order $n_{e}(0) \leq n_{e}(1)$ is found here due to the fact that $p \mu>\gamma$ in our parameter setting, and this is corresponding with the result in Proposition 3.2. Figure 6 shows the thresholds with respect to vacation rate $\gamma$ and blocked customers prefer to join the system with a larger vacation rate $\gamma$, since the server can be available with a larger proportion of time and seek them. Moreover, the value of the difference $n_{e}(0)-n_{e}(1)$ is negative 


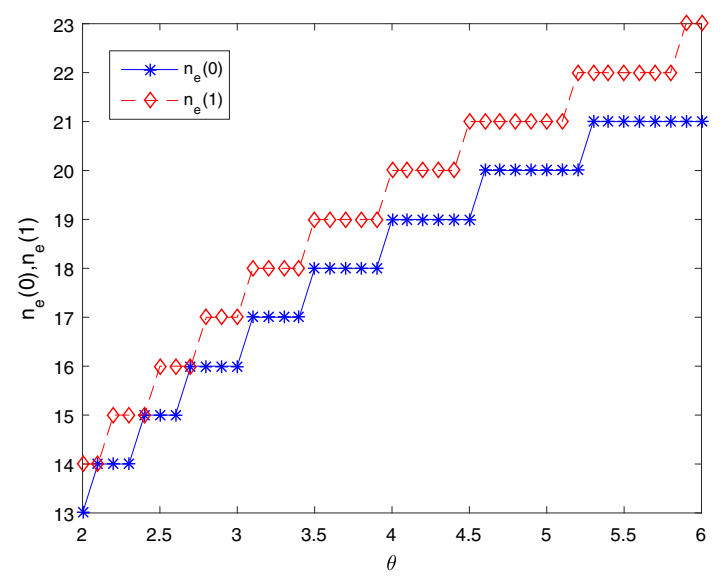

FiguRE 5. Thresholds in the fully observable case vs. $\theta$ for $\lambda=1, \mu=3, \gamma=0.5, p=0.8$.

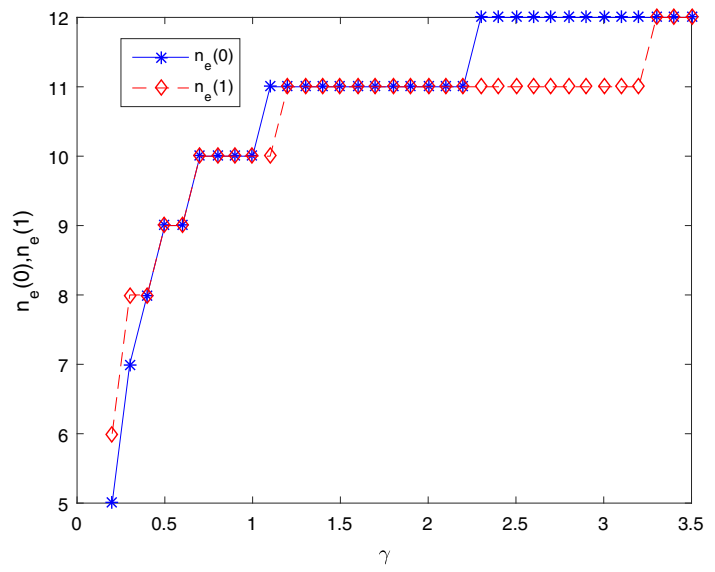

Figure 6. Thresholds in the fully observable case $v s$. $\gamma$ for $\lambda=0.5, \mu=1.2, \theta=1.5, p=0.8$.

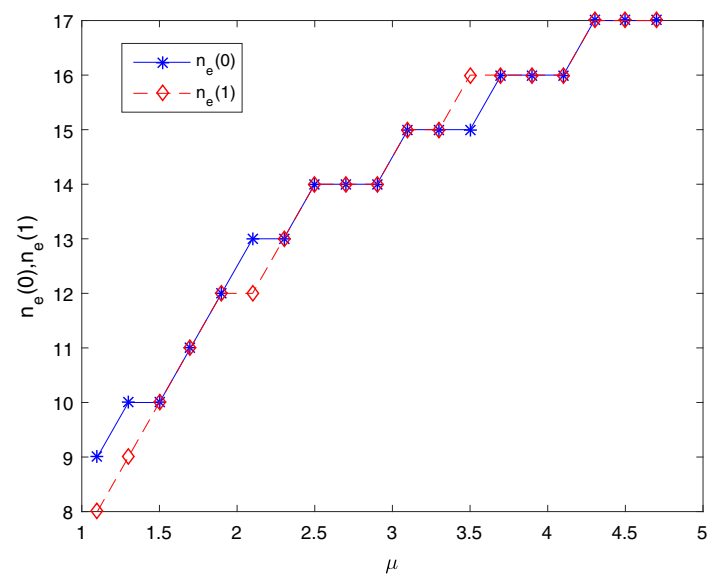

FiguRE 7. Thresholds in the fully observable case vs. $\mu$ for $\lambda=1, \theta=1.5, \gamma=1.5, p=0.6$. 


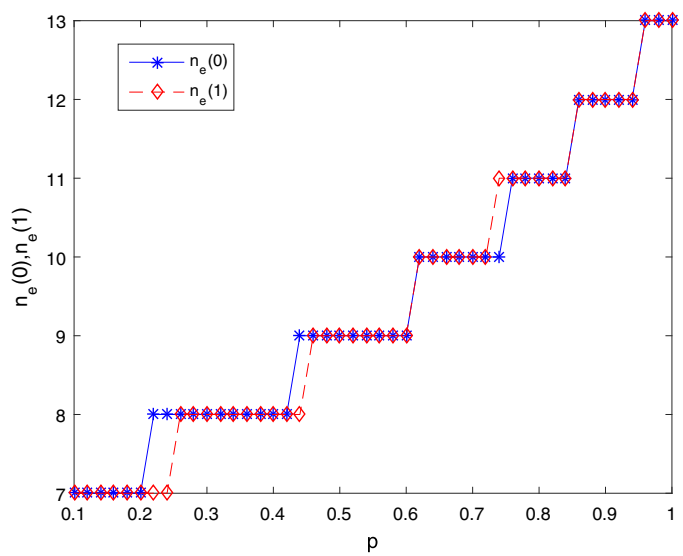

Figure 8. Thresholds in the fully observable case vs. $p$ for $\lambda=1, \mu=1.5, \gamma=1, \theta=1.5$.

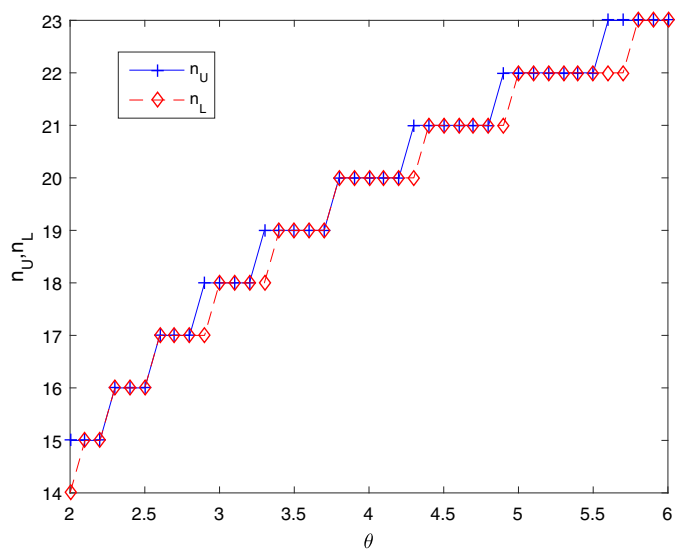

Figure 9 . The value of $n_{U}, n_{L}$ in the almost observable case with respect to $\theta, \lambda=1, \mu=$ $3, \gamma=0.5, p=0.8$.

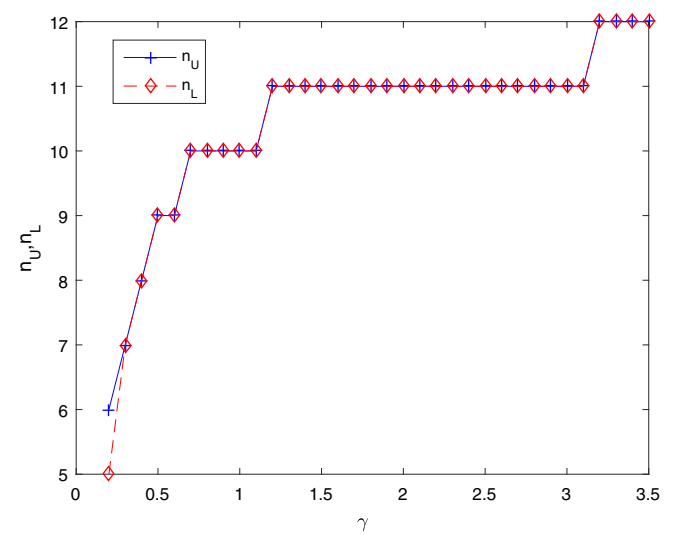

Figure 10 . The value of $n_{U}, n_{L}$ in the almost observable case with respect to $\gamma, \lambda=0.5, \mu=$ $1.2, \theta=1.5, p=0.8$. 


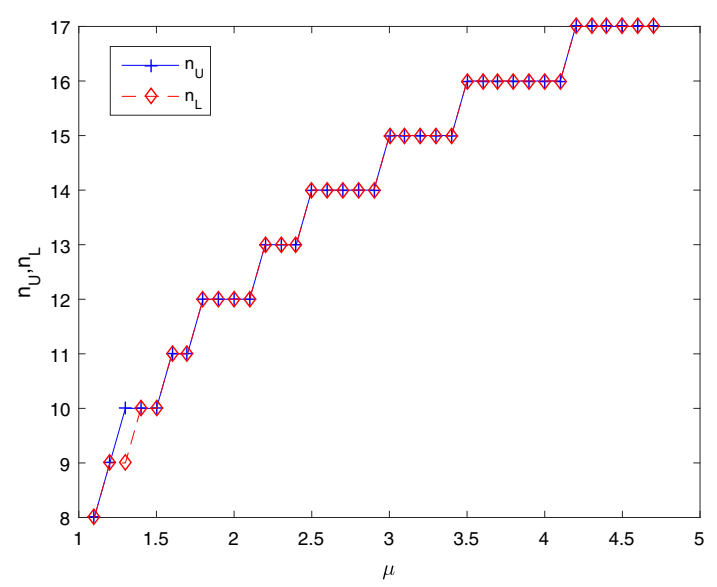

Figure 11. The value of $n_{U}, n_{L}$ in the almost observable case with respect to $\mu, \lambda=1, \theta=$ $1.5, \gamma=1.5, p=0.6$.

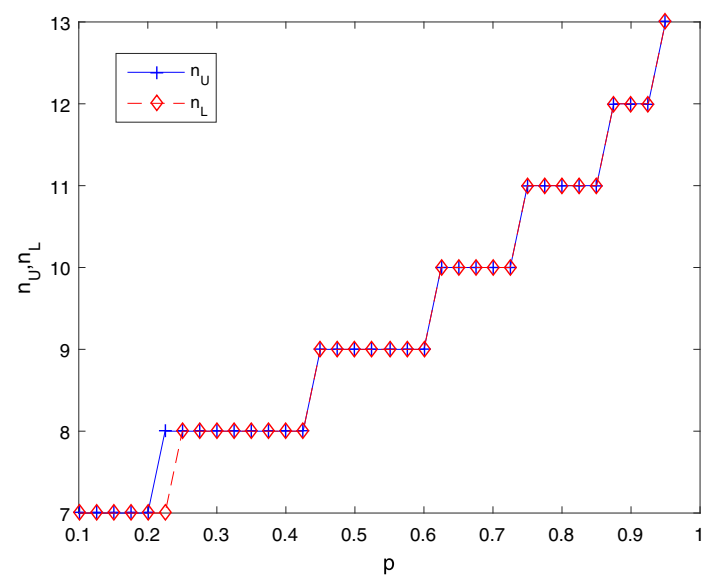

Figure 12. The value of $n_{U}, n_{L}$ in the almost observable case with respect to $p, \lambda=1, \mu=$ $1.5, \gamma=1, \theta=1.5$.

first and then be positive which can be explained by Proposition 3.2. With the increasing of $\gamma$, the server under vacation is more likely to be available than a busy server, thus arrivals prefer to join the system when $I(t)=0$ later. The numerical examples shown in Figure 7 indicates that customers' joining willingness increase in service rate $\mu$. For a relatively small $\mu$ which satisfies $p \mu \leq \gamma$, we have $n_{e}(0) \geq n_{e}(1)$, to the contrary, $n_{e}(0)<n_{e}(1)$ for a larger $\mu$. Finally, in Figure 8, the thresholds vary with the parameter $p$ (the probability that the server remains available after a service completion). Similarly, customers in the orbit are more likely be searched with a larger $p$ and the order of the two thresholds can be also explained by Proposition 3.2.

Figures 9-12 show the variation tendency of the upper and lower bounds of the threshold $n_{e}: n_{U}$ and $n_{L}$ in the almost observable case. In all figures, we observe that $n_{L} \leq n_{U}$. By comparing the thresholds under two information cases with same parameters, we find that the thresholds in the almost observable case in the middle range of the two thresholds in the fully observable case. That is to say, $\min \left\{n_{e}(0), n_{e}(1)\right\} \leq n_{L} \leq n_{U} \leq$ $\max \left\{n_{e}(0), n_{e}(1)\right\}$ holds in our model. What's more, the variation tendency can be explained similarly with these in the fully observable case. 


\section{Conclusions}

In this paper, we analyzed blocked customers' equilibrium joining behavior in a single-server constant retrial queue with Bernoulli vacations, where arrivals can observe the number of customers in the orbit. The blocked customers in the orbit was searched by the server according to their arrivals order when the server is idle. Accordingly, we obtained the threshold equilibrium strategies of blocked customers in different information levels. In the majority of queueing studies with vacations, customers are more willing to join the system when server is busy than it is under vacation, while in this paper, there exists a trade-off between the two states due to the existence of Bernoulli schedule. We found that they only care about the server's transfer rate of being available. To the best of authors' knowledge, this is the first work that studies customers' equilibrium behavior in constant retrial queueing systems with Bernoulli vacations based on game theory. The influences of some important parameters on the equilibrium thresholds has been investigated extensively. In the future, the equilibrium analysis could be extended to more general situations with Bernoulli schedule, such as the $M / G / 1$ retrial queueing system, a same model with finite buffer in the orbit, or a similar model but with a general searching time distribution.

Acknowledgements. This work was supported in part by the National Natural Science Foundation of China (Grant Nos. 71871008, 71571014) and the Fundamental Research Funds for the Central Universities (No. 2019YJS196).

\section{REFERENCES}

[1] J.R. Artalejo, A. Gómez-Corral and M.F. Neuts, Analysis of multiserver queues with constant retrial rate. Eur. J. Oper. Res. 135 (2001) 569-581.

[2] K. Avrachenkov and U. Yechiali, Retrial networks with finite buffers and their application to internet data traffic. Prob. Eng. Inf. Sci. 22 (2008) 519-536.

[3] K. Avrachenkov and U. Yechiali, On tandem blocking queues with a common retrial queue. Comput. Oper. Res. 37 (2010) $1174-1180$.

[4] A. Burnetas and A. Economou, Equilibrium customer strategies in a single server Markovian queue with setup times. Queueing Syst. 56 (2007) 213-228.

[5] J.W. Cohen, Basic problems of telephone traffic theory and the influence of repeated calls. Philips Telecommun. Rev. 18 (1957) 49-100.

[6] A. Economou and S. Kanta, Equilibrium balking strategies in the observable single-server queue with breakdowns and repairs. Oper. Res. Lett. 36 (1957) 696-699.

[7] A. Economou and S. Kanta, Equilibrium customer strategies and social-profit maximization in the single-server constant retrial queue. Naval Res. Logist. 58 (2011) 107-122.

[8] N.M. Edelson and K. Hildebrand, Congestion tolls for Poisson queuing processes. Econometrica 43 (1975) 81-92.

[9] G. Falin, A survey of retrial queues. Queueing Syst. 7 (1990) 127-167.

[10] G.I. Falin and J.G. Templeton, Retrial Queues. Chapman and Hall, London (1997).

[11] G. Fayolle, A simple telephone exchange with delayed feedbacks. Proceeding of the International Seminar on Teletraffic Analysis and Computer Performance Evaluation, Amsterdam (1986) 245-253.

[12] R. Hassin, Rational Queueing. Chapman \& Hall/CRC Series in Operations Research (2016).

[13] R. Hassin and M. Haviv, To Queue or Not to Queue: Equilibrium Behavior in Queueing Systems. Kluwer Academic Publishers, Netherlands (2003).

[14] J. Keilson and L.D. Servi, Oscillating random walk models for GI/G/1 vacation systems with Bernoulli schedules. J. Appl. Prob. 23 (1986) 790-802.

[15] B.K. Kumar and D. Arivudainambi, The M/G/1 retrial queue with Bernoulli schedules and general retrial times. Comput. Math. Appl. 43 (2002) 15-30.

[16] B.K. Kumar and S.P. Madheswari, Analysis of an M/M/N queue with Bernoulli service schedule. Int. J. Oper. Res. 5 (2009) 48-72.

[17] B.K. Kumar, D. Arivudainambi and A. Vijayakumar, On the $\mathrm{M}(\mathrm{x}) / \mathrm{G} / 1$ retrial queue with Bernoulli schedules and general retrial times. Asia-Pac. J. Oper. Res. 19 (2002) 177.

[18] B.K. Kumar, R. Rukmani and V. Thangaraj, An M/M/C retrial queueing system with Bernoulli vacations. J. Syst. Sci. Syst. Eng. 18 (2009) 222.

[19] B.K. Kumar, R. Rukmani and S.A. Lakshmi, Performance analysis of an M/G/1 queueing system under Bernoulli vacation schedules with server setup and close down periods. Comput. Ind. Eng. 66 (2013) 1-9.

[20] V.G. Kulkarni, A game theoretic model for two types of customer's competing for service. Oper. Res. Lett. 2 (1983) 119-122. 
[21] T. Li, L. Zhang and S. Gao, An M/G/1 retrial queue with balking customers and Bernoulli working vacation interruption. Qual. Technol. Quant. Manage. 16 (2018) 511-530.

[22] J. Liu and J. Wang, Strategic joining rules in a single server Markovian queue with Bernoulli vacation. Oper. Res. 17 (2017) 413-434.

[23] M. Martin and J. Artalejo, Analysis of an M/G/1 queue with two types of impatient units. Adv. Appl. Prob. 27 (1995) 840-861.

[24] P. Naor, The regulation of queue size by levying tolls. Econometrica 37 (1969) 15-24.

[25] N. Tian and Z.G. Zhang, Vacation Queueing Models: Theory and Applications. Springer Science \& Business Media 93 (2006).

[26] J. Wang and F. Zhang, Strategic joining in M/M/1 retrial queues. Eur. J. Oper. Res. 37 (2013) 76-87.

[27] F. Wang, J. Wang and F. Zhang, Strategic behavior in the single-server constant retrial queue with individual removal. Qual. Technol. Quant. Manage. 12 (2015) 325-342.

[28] J. Wang, X. Zhang and P. Huang, Strategic behavior and social optimization in the constant retrial queue with N-policy. Eur. J. Oper. Res. 256 (2017) 841-849.

[29] Z. Zhang, J. Wang and F. Zhang, Equilibrium customer strategies in the single-server constant retrial queue with breakdowns and repairs. Math. Probl. Eng. 2014 (2014) 379572.

[30] W. Zhou, Analysis of a single-server retrial queue with FCFS orbit and Bernoulli vacation. Appl. Math. Comput. 161 (2005) 353-364. 